\title{
Comparing SWAS and ISO observations of water in outflows
}

\author{
M. Benedettini ${ }^{1}$, S. Viti ${ }^{2}$, T. Giannini ${ }^{3}$, B. Nisini ${ }^{3}$, P. F. Goldsmith ${ }^{4}$, and P. Saraceno ${ }^{1}$ \\ ${ }^{1}$ CNR-Istituto di Fisica dello Spazio Interplanetario, Area di Ricerca di Tor Vergata, via del Fosso del Cavaliere 100, \\ 00133 Roma, Italy \\ 2 Department of Physics and Astronomy, UCL, Gower St., London WC1E 6BT, UK \\ 3 INAF-Osservatorio Astronomico di Roma, via Frascati 33, 00040 Monte Porzio, Italy \\ ${ }^{4}$ National Astronomy and Ionosphere Center, Department of Astronomy, Cornell University, Space Sciences Building, Ithaca, \\ NY 14853-6801, USA
}

Received 24 April 2002 / Accepted 27 August 2002

\begin{abstract}
We present a detailed comparison of SWAS and ISO observations of $\mathrm{H}_{2} \mathrm{O}$ emission in a sample of outflows. By taking into consideration the different methods used to derive the fractional water abundance, we find that, despite the initial apparent discrepancies, there is satisfactory agreement between ISO and SWAS results for the outflows observed by both satellites. Such an agreement is reached by assuming that most of the detected water comes from a single region smaller than both the ISO and SWAS beam apertures and considering temperatures higher $(T>100 \mathrm{~K})$ and density lower $\left(n\left(\mathrm{H}_{2}\right)<2 \times 10^{6} \mathrm{~cm}^{-3}\right)$ than previously adopted. The ratio between the integrated flux of the ortho- $\mathrm{H}_{2} \mathrm{O} 2_{12}-1_{01}$ line at $179.5 \mu \mathrm{m}$ observed by ISO and the fundamental ortho- $\mathrm{H}_{2} \mathrm{O} 1_{10}-1_{01}$ line at $538.3 \mu \mathrm{m}$ observed by SWAS is always smaller (never by more than a factor of 7) than the expected ratio. We propose that the fundamental ortho- $\mathrm{H}_{2} \mathrm{O}$ transition has a contribution from a cooler gas component to which SWAS is sensitive but which is not traced by ISO. We conclude that, while ISO and SWAS observations are consistent with chemical models predicting a significant enhancement of water vapor abundance in outflow regions, the explanation for the low water abundance derived from SWAS observations of the cold ISM is still unclear.
\end{abstract}

Key words. ISM: jets and outflows - ISM: abundances - ISM: molecules

\section{Introduction}

Water is an important reservoir of the elemental oxygen in the Interstellar Medium (ISM) and it is thought to provide a major source of cooling. However, due to terrestrial atmospheric absorption, observing water in the ISM from the ground has always been a challenge. It was not until the advent of Infrared Space Observatory (ISO) and more recently Submillimeter Wave Astronomy Satellite (SWAS) that direct detection of thermal water in warm and cold gas has been possible.

The Long Wavelength Spectrometer (LWS) on the ISO satellite has been used to observe $\mathrm{H}_{2} \mathrm{O}$ in a variety of regions: $\mathrm{M}$ and K giants (Tsuji 2001), outflows (Giannini et al. 2001, hereafter GNL), Mira variables (Truong-Bach et al. 1999), etc. One of the advantages of the LWS was the large spectral range available, 43-196.9 $\mu \mathrm{m}$, which is rich in rotational lines of water vapor, in both the ortho and para form. This gave the possibility, for the first time, to derive the water abundance by multiline observations in a variety of regions in the dense ISM. The price for the large spectral range was paid, however, in terms of the low spectral resolution $\left(\sim 1000 \mathrm{~km} \mathrm{~s}^{-1}\right.$ in the grating configuration), which prevented to spectrally resolve the lines.

Send offprint requests to: $\mathrm{M}$. Benedettini,

e-mail: milena@ifsi.rm.cnr.it
SWAS, on the other hand, has conducted pointed observations in the low-lying transitions of five molecular species, including the fundamental ortho- $\mathrm{H}_{2} \mathrm{O} 1_{10}-1_{01}$ transition at $538.3 \mu \mathrm{m}$. Its high spectral resolution $\left(\sim 1 \mathrm{~km} \mathrm{~s}^{-1}\right)$ allows measurement of line profiles sufficiently well to distinguish between the emission from outflows and shocked regions and that from more quiescent regions. However, since SWAS observes only one water transition, the estimated water abundance may not be very accurate (see Sect. 2).

ISO and SWAS observations have placed strict constraints on the fractional abundance of water in both the warm and cold ISM. For cold quiescent gas, SWAS has found a clear discrepancy between observations and model predictions. While the latter always predict fractional abundances of water $\geq 10^{-7}$ with respect to $\mathrm{H}_{2}$, observations have indicated that, on average, water is more than two orders of magnitude less abundant (Bergin et al. 2000). Both ISO and SWAS observations of warm gas, in particular of outflow regions, show an enhancement of the water fractional abundance with respect to quiescent regions. From the ISO data a fractional abundance of water of $10^{-5}-10^{-4}$ has been derived in outflows originating from low-mass protostars (GNL and references therein) and $\sim 5 \times 10^{-4}$ for the high-mass star formation region Orion BN/KL (Harwit et al. 1998; Cernicharo et al. 1999). A high 
value $\left(X\left(\right.\right.$ ortho- $\left.\left.\mathrm{H}_{2} \mathrm{O}\right)=3.5 \times 10^{-4}\right)$ toward Orion $\mathrm{BN} / \mathrm{KL}$ has been found also by SWAS (Melnick et al. 2000a) while for 3 other low-mass outflows SWAS observations indicate an abundance $\lesssim 10^{-6}$ (Neufeld et al. 2000, hereafter NSA). This latter value is lower than what is expected in these regions, where the presence of shocks should enhance the water fractional abundance to values of more than $10^{-4}$ (Draine et al. 1993; Kaufman \& Neufeld 1996).

A more detailed study of water in outflows regions is clearly needed. We therefore decided to examine a sample of outflows observed by both the ISO and SWAS satellites to compare in more detail their results. We report our findings in this paper.

\section{ISO and SWAS water abundance derivations}

Before comparing ISO and SWAS data, it is important to be aware of the differences between the instruments on board these two satellites and between the appropriate methods of deriving the fractional abundance of water vapor.

SWAS is tuned to observe the ground state ortho- $\mathrm{H}_{2} \mathrm{O}$ transition at $538.3 \mu \mathrm{m}$, having $h v / k=27 \mathrm{~K}$, which can thus be highly populated at low temperatures. On the other hand, ISO can detect several high energy transitions which become more populated as the temperature increases. Consequently, ISO is more sensitive to warm water vapor while SWAS is more sensitive to cooler gas. The SWAS beam is quite large (3.3×4.5) and typically encompasses the entire outflow; the ISO-LWS beam is smaller $\left(\sim 75^{\prime \prime}\right)$, and for the extended outflows, different pointings were required to cover the entire outflow region.

ISO-LWS has detected a large number of water transitions in several outflows, and always in conjunction with high-J CO rotational lines. The most widely used method to derive the fractional water abundance assumes that $\mathrm{CO}$ and $\mathrm{H}_{2} \mathrm{O}$ come from the same component of the gas: the density and temperature derived from the $\mathrm{CO}$ lines are then used to fit the water transitions and to derive the water fractional abundance (the standard $\mathrm{CO} / \mathrm{H}_{2}$ ratio of $10^{-4}$ is adopted). The fitting is usually done by means of a radiative transfer model and statistical equilibrium equations under the assumption of Large Velocity Gradient (LVG) conditions and plane parallel geometry (see Giannini et al. 1999 for the CO model and Nisini et al. 1999 for the $\mathrm{H}_{2} \mathrm{O}$ model). The validity of the LVG assumption is confirmed by the broad line width $\left(\gtrsim 20 \mathrm{~km} \mathrm{~s}^{-1}\right)$ shown in the SWAS water spectra (NSA and Fig. 1).

SWAS observations and analyses of 3 outflows driven by low-mass YSO are reported by NSA. They assume that the water emission comes from the large scale molecular outflow and they use the mass, mean density and mean temperature of the outflow together with the integrated flux of the fundamental transition of the ortho- $\mathrm{H}_{2} \mathrm{O}$ to obtain the water fractional abundance, through the following equation:

$\frac{n\left(\mathrm{o}-\mathrm{H}_{2} \mathrm{O}\right)}{n\left(\mathrm{H}_{2}\right)}=2.0 \times 10^{-5} \frac{d^{2}}{n_{5} M q_{-11}} \int T_{\mathrm{A}}^{*} \mathrm{~d} v$,

where $d$ is the distance in $\mathrm{kpc}, n_{5}$ is the $\mathrm{H}_{2}$ density in units of $10^{5} \mathrm{~cm}^{-3}, M$ is the outflow mass in solar masses, $q_{-11}$ is the rate

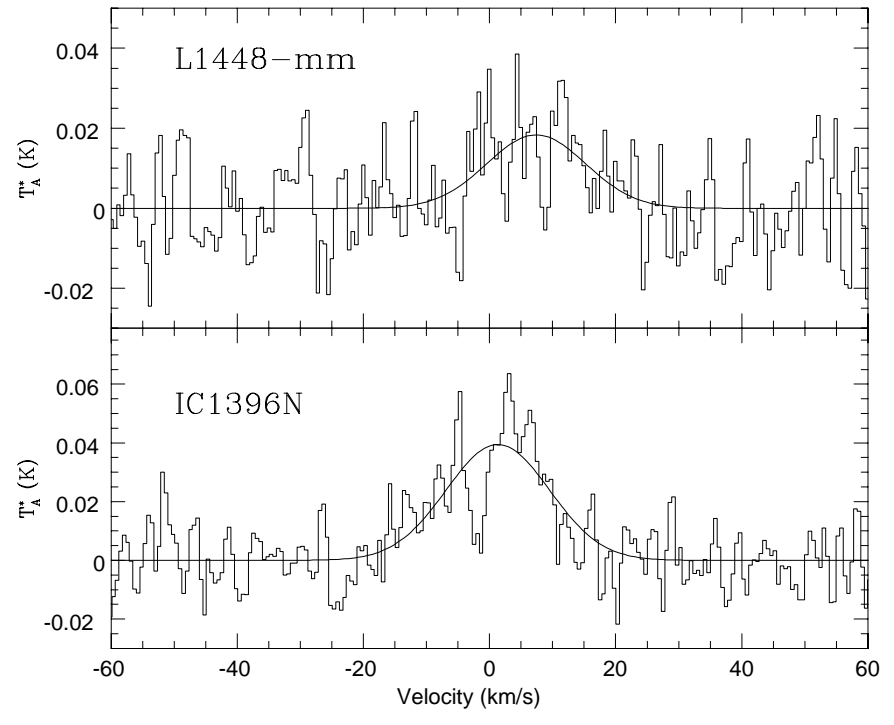

Fig. 1. SWAS detection of the $538.3 \mu \mathrm{m}$ water transition in L1448-mm (upper panel) and IC 1396N (lower panel).

coefficient of the collisional excitation with $\mathrm{H}_{2}$ for the transition $1_{10}-1_{01}$ in units of $10^{-11} \mathrm{~cm}^{3} \mathrm{~s}^{-1}$. The physical parameters used in the equation must be representative of the mean conditions of the outflow; however, the estimate of the parameters depends on which molecule and transitions are used to derive them. NSA used the low- $J$ CO lines, tracing the large scale molecular outflow, to derive the emitting mass and molecules such as $\mathrm{CS}$ and $\mathrm{H}^{13} \mathrm{CO}^{+}$, tracing the denser gas closer to the central protostar, to derive the temperature and the density. The shortcomings of this method, as pointed out by NSA, are that the density and the temperature derived from molecules such as $\mathrm{CS}$ and $\mathrm{NH}_{3}$ is applied uniformly over the entire outflow mass traced by low-J CO line wings. The $538 \mu \mathrm{m}$ transition has a high critical density $\left(\sim 10^{9} \mathrm{~cm}^{-3}\right)$ and it is expected to have a high optical depth, therefore trapping is important. Nevertheless, if the density of the medium is much smaller than the effective critical density (as it is in the outflow regions), collisional excitation always results in a photon that escapes the cloud, meaning that the transition may be optically thick but effectively thin. However, for very high densities, the $\mathrm{H}_{2} \mathrm{O}$ abundance derived from Eq. (1) can be considered to be a lower limit.

\section{Data analysis and results}

In Table 1 we summarize the SWAS observations for four outflows that have also been observed by ISO: the coordinates are listed in Cols. 2 and 3, the integration time in Col. 4 and the line flux, integrated over the line profile, in Col. 5. Two of these objects have already been published by NSA, i.e. NGC 1333-IRAS 4 and L1157-mm; the new data of L1448-mm and IC $1396 \mathrm{~N}$ are shown in Fig. 1. All SWAS data were obtained in the nodded observational mode (Melnick et al. 2000b) with nodding offset between $1^{\circ}$ and $4^{\circ}$, and reduced using the standard SWAS pipeline. For L1448-mm, ISO data show strong water emission while SWAS detects only a very weak signal (see Fig. 1, top panel); both ISO and SWAS have detected 
Table 1. Coordinates, integration time and total line flux of the SWAS observations.

\begin{tabular}{|c|c|c|c|c|}
\hline source & $\begin{array}{l}\alpha(2000) \\
\text { h m s }\end{array}$ & $\begin{array}{c}\delta(2000) \\
\circ, \quad, \quad\end{array}$ & $\begin{array}{c}t_{\text {int }} \\
\text { hours }\end{array}$ & $\begin{array}{c}\int T_{\mathrm{A}}^{*} \mathrm{~d} v \\
\mathrm{~K} \mathrm{~km} \mathrm{~s}^{-1}\end{array}$ \\
\hline L1448-mm & 032530.5 & 304543 & 31.47 & $0.37 \pm 0.04$ \\
\hline NGC 1333-IRAS 4 & 032911.1 & 311320 & 27.63 & $1.27^{\dagger}$ \\
\hline L1157-mm & 203906.5 & 680214 & 42.12 & $1.34^{\dagger}$ \\
\hline IC $1396 \mathrm{~N}$ & 214042.3 & 581610 & 37.15 & $0.78 \pm 0.04$ \\
\hline
\end{tabular}

${ }^{\dagger}$ From Neufeld et al. (2000) where they are reported without errors.

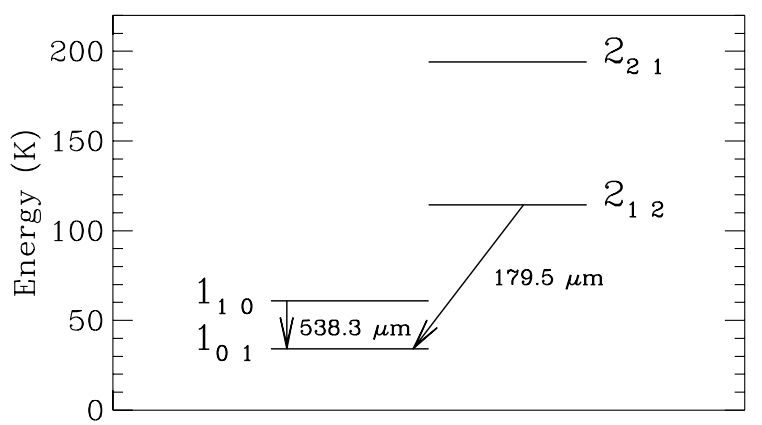

Fig. 2. Ortho- $\mathrm{H}_{2} \mathrm{O}$ energy level diagram.

water in NGC 1333-IRAS 4 and IC 1396N; finally, SWAS has detected a strong water transition in L1157-mm while ISO has only detected a few weak lines.

Given the relatively large ISO-LWS beam $\left(\sim 75^{\prime \prime}\right)$ we cannot be sure where the high- $J \mathrm{CO}$ and $\mathrm{H}_{2} \mathrm{O}$ transitions detected by LWS in outflow regions come from. In particular, the LWS lines observed in the on-source pointings have been widely interpreted by a non-dissociative, low velocity C-shock (GNL, Nisini et al. 1999; Molinari et al. 2000) associated with the base of the outflow, although some of the emission could be also produced in the inner warm part of the circumstellar envelope for the most luminous sources (Ceccarelli et al. 1999). On the other hand, it seems clear from the SWAS spectra of the orthowater fundamental transition in outflows (see Fig. 1 and NSA) that this line is emitted from a shocked region; this is likely to be the same region emitting the high- $J \mathrm{CO}$ and $\mathrm{H}_{2} \mathrm{O}$ lines detected by ISO. We therefore assume in our analysis that the water observed by SWAS comes from the same gas component observed by ISO and we use the LVG approximation for modeling the emission. In order to quantitatively compare the ISO observations with the SWAS ortho- $\mathrm{H}_{2} \mathrm{O} 1_{10}-1_{01}$ transition at $538.3 \mu \mathrm{m}$ we consider the ortho- $\mathrm{H}_{2} \mathrm{O} \quad 2_{12}-1_{01}$ line at $179.5 \mu \mathrm{m}$ since these are both low-lying resonance lines and they are the two channels through which the excited molecule can decay to the fundamental level (see Fig. 2). In the LVG approximation the ratio between two transitions of the same species depends on the temperature and density of the gas, and the ratio between the column density and the linewidth of each transition. We calculate the expected ratio between the intensity of the $179.5 \mu \mathrm{m}$ and $538.3 \mu \mathrm{m}$ transitions, $R(179 / 538)$, using our LVG model neglecting radiative excitation by warm dust (Nisini et al. 1999) and assuming the same filling factor for the two lines. In Fig. 3 we show the ratio calculated by adopting
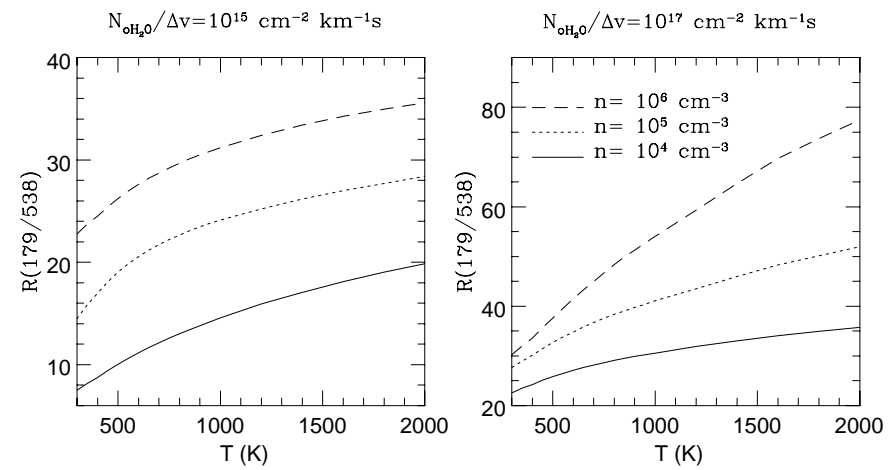

Fig. 3. Theoretical ratio between the intensity of the $179.5 \mu \mathrm{m}$ and $538.3 \mu \mathrm{m}$ lines vs. temperature for different densities $\left(10^{4} \mathrm{~cm}^{-3}\right.$ solid line, $10^{5} \mathrm{~cm}^{-3}$ dotted line and $10^{6} \mathrm{~cm}^{-3}$ dashed line) and $N\left(\mathrm{o}-\mathrm{H}_{2} \mathrm{O}\right) / \Delta v\left(10^{15} \mathrm{~cm}^{-2} \mathrm{~km}^{-1} \mathrm{~s}\right.$ left panel and $10^{17} \mathrm{~cm}^{-2} \mathrm{~km}^{-1} \mathrm{~s}$ right panel). The same filling factor is assumed.

the typical physical conditions derived from ISO for outflow regions, $300<T<2000 \mathrm{~K}, 10^{4}<n\left(\mathrm{H}_{2}\right)<10^{6} \mathrm{~cm}^{-3}, 10^{15}<$ $N\left(\mathrm{o}-\mathrm{H}_{2} \mathrm{O}\right) / \Delta v<10^{17} \mathrm{~cm}^{-2} \mathrm{~km}^{-1}$ s. $R(179 / 538)$ increases with temperature, from $\sim 10$ to $\sim 80$, indicating that for the warm gas the $179.5 \mu \mathrm{m}$ line is the preferential channel to decay to the ground state.

To calculate the observed ratio between the flux density of the $179 \mu \mathrm{m}$ and $538 \mu \mathrm{m}$ lines, we convert the integrated antenna temperature of the SWAS line into total line flux by using the formula:

$F\left(\mathrm{~W} \mathrm{~cm}^{-2}\right)=9.2 \times 10^{-29} \frac{v}{A_{\mathrm{e}}} \int T_{\mathrm{A}}^{*} \mathrm{~d} v\left(\mathrm{~K} \mathrm{~km} \mathrm{~s}^{-1}\right)$

where $v$ is the line frequency in $\mathrm{Hz}$ and $A_{\mathrm{e}}=1.9 \times 10^{3} \mathrm{~cm}^{2}$ is the effective area of the antenna. This formula is valid when the size of the source is small compared to the beam of the antenna, indeed the high temperature component of the outflow is likely to fill only a small fraction of the SWAS beam.

We also calculate the ortho-water fractional abundance from the fundamental transition measured by SWAS using Eq. (1) and assuming the density, temperature and mass derived from the high- $J$ CO lines observed by ISO and the excitation rate coefficient by Green et al. (1993). The Green et al. coefficients are calculated for collision with Helium and are then scaled for the $\mathrm{H}_{2}$ assumed to be in the $J=0$ rotational level. However, at the adopted temperatures of $\sim 1000 \mathrm{~K}$, a significant fraction of $\mathrm{H}_{2}$ can be excited in the higher rotational levels, as is actually observed for example in L1448-mm (Nisini et al. 1999, 2000). Phillips et al. (1996) calculated collisional rate coefficients for excitation by $\mathrm{H}_{2}(J=0)$ and $\mathrm{H}_{2}(J=1)$ for temperatures $<140 \mathrm{~K}$, finding that, especially for ortho- $\mathrm{H}_{2} \mathrm{O}$, the rates are significantly higher than those of Green et al. If this trend continues at higher temperatures, our water fractional abundances may be overestimated.

In Table 2 we summarize the results: the distance of the source is reported in Col. 2; the density, mass and temperature (Cols. 3 to 5) are the values, averaged along the outflow, derived by ISO from the high- $J$ CO lines; in Col. 6 we give the excitation coefficient for the water transition $1_{10}-1_{01}$; the observed and predicted ratios $R(179 / 538)$ are given in Col. 7 and 
Table 2. Values for the physical quantities of the observed outflows. See text for references.

\begin{tabular}{|l|c|c|c|c|c|c|c|c|c|}
\hline \hline source & $d$ & $n\left(\mathrm{H}_{2}\right)$ & Mass & $T$ & $q$ & \multicolumn{2}{|c|}{$R(179.5 / 538.3)$} & $X\left(\mathrm{o}-\mathrm{H}_{2} \mathrm{O}\right)$ & $X\left(\mathrm{H}_{2} \mathrm{O}\right)$ \\
& $\mathrm{kpc}$ & $10^{5} \mathrm{~cm}^{-3}$ & $M_{\odot}$ & $\mathrm{K}$ & $10^{-11} \mathrm{~cm}^{3} \mathrm{~s}^{-1}$ & obs. & mod. & SWAS & ISO \\
\hline L1448-mm & 0.35 & 0.4 & 0.015 & 900 & 11.125 & 10 & 25 & $1 \times 10^{-5}$ & $2 \times 10^{-4}$ \\
NGC 1333-IRAS 4 & 0.35 & 0.8 & 0.013 & 1300 & 12.650 & 4 & 27 & $2 \times 10^{-5}$ & $1 \times 10^{-4}$ \\
L1157-mm & 0.44 & 3 & 0.004 & 600 & 9.065 & 4 & 6 & $5 \times 10^{-5}$ & $5 \times 10^{-5}$ \\
IC 1396N & 0.75 & 20 & 0.003 & 1400 & 12.900 & 10 & 37 & $1 \times 10^{-5}$ & $1 \times 10^{-4}$ \\
IC 1396N & 0.75 & 20 & 0.030 & 300 & 5.885 & 10 & 27 & $3 \times 10^{-6}$ & $3 \times 10^{-5}$ \\
\hline
\end{tabular}

in Col. 8 respectively; the fractional abundance of ortho-water derived from the SWAS transition is reported in Col. 9 while in Col. 10 we report the (ortho+para) water fractional abundance derived from the ISO data.

\section{1. $L 1448-m m$}

L1448-mm is a deeply embedded, very young low mass protostellar object $\left(L \sim 10 L_{\odot}\right)$ located in the core of the L1448 dense cloud (distance $350 \mathrm{pc}$ ). This source drives a highly collimated bipolar outflow (Bachiller et al. 1990) mapped by different tracers (such as $\mathrm{CO}, \mathrm{H}_{2}, \mathrm{SiO}$ ) which reveal a complex structure.

The case of the L1448-mm outflow may constitute an anomaly: both ISO and SWAS have extensively observed this source but while the ISO-LWS spectrum reveals strong water lines and a high fractional abundance of water has been derived (Nisini et al. 2000), SWAS detects only weak emission of the fundamental water transition (see Fig. 1).

The theoretical $R(179 / 538)$ calculated for the physical conditions derived from ISO is $\sim 25$ (see Table 2). The flux of the $179 \mu \mathrm{m}$ line averaged along the flow is $1.1 \times 10^{-19} \mathrm{~W} \mathrm{~cm} \mathrm{~cm}^{-2}$ (Nisini et al. 2000); therefore the expected flux of the $538.3 \mu \mathrm{m}$

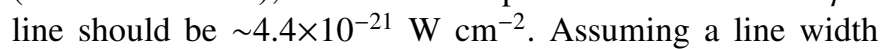
of $15 \mathrm{~km} \mathrm{~s}^{-1}$ (which is the value we can roughly infer from the SWAS profile) for the $538.3 \mu \mathrm{m}$ line, the main brightness temperature corresponding to a flux of $4.4 \times 10^{-21} \mathrm{~W} \mathrm{~cm}^{-2}$ is $\sim 0.012 \mathrm{~K}$, slightly higher than the spectral noise that SWAS reaches in 30 hours of integration time. The $538.3 \mu \mathrm{m}$ transition should therefore have been detected toward L1448-mm. In fact, although only a $2 \sigma$ detection in terms of the peak temperature of the $538.3 \mu \mathrm{m}$ line is reported (see Fig. 1, top panel), if we consider the integrated line intensity a clear line emission $(\sim 10 \sigma)$ is detected. From this detection we derive an observed $R(179 / 538)$ of $\sim 10$, only a factor of 2.5 smaller than the theoretical ratio. We conclude that the agreement is satisfactory. Given the large extent of the L1448-mm outflow, three different LWS pointings were needed to map the entire outflow. Consequently, to derive the water fractional abundance using Eq. (1), we employ the physical parameters derived from the average of ISO on source and on lobes measurements (Nisini et al. 2000), namely $n\left(\mathrm{H}_{2}\right)=4 \times 10^{4} \mathrm{~cm}^{-3}, T=900 \mathrm{~K}$ and $M=$ $1.5 \times 10^{-2} M_{\odot}$. The fractional abundance for ortho-water derived from the SWAS transition is $10^{-5}$, one order of magnitude less than the mean abundance derived from ISO data $\left(2 \times 10^{-4}\right)$.

\subsection{NGC 1333-IRAS 4}

NGC 1333 is a low mass star formation region lying within the L1450 molecular cloud complex at distance of $350 \mathrm{pc}$. Both ISO (Ceccarelli et al. 1999, GNL) and SWAS (NSA) have detected water towards the embedded central source $\left(L \sim 14 L_{\odot}\right)$ as well as from the outflow lobes.

The expected $R(179 / 538)$ value is $\sim 27$; however the observed ratio is smaller i.e. the intensity of the line observed by SWAS is a factor of $\sim 7$ larger than predicted by the LVG model. We will discuss this point in the next section.

NSA find a fractional ortho- $\mathrm{H}_{2} \mathrm{O}$ abundance of $1.6 \times 10^{-6}$. We recalculate the abundance from the SWAS line assuming the physical parameters derived from ISO data. Averaging the value derived by GNL on source and on the red lobe we assume that $n\left(\mathrm{H}_{2}\right)=8 \times 10^{4} \mathrm{~cm}^{-3}, T=1300 \mathrm{~K}$ and $M=1.3 \times 10^{-2} M_{\odot}$. We find $X\left(\mathrm{o}-\mathrm{H}_{2} \mathrm{O}\right) \approx 2 \times 10^{-5}$ one order of magnitude higher than the value derived by NSA. This difference may be due to the fact that NSA assume the molecular outflow mass derived from the low- $J$ CO line and attributed to that emitting material values for the density $\left(5 \times 10^{6} \mathrm{~cm}^{-3}\right)$ and temperature $(80 \mathrm{~K}) \mathrm{de}$ rived from $\mathrm{H}_{2} \mathrm{CO}$ line ratios in a region $20^{\prime \prime}$ in size around the exciting source. These values are probably not representative of the mean density and temperature of the overall molecular outflow traced by the low- $J$ CO line. Note that our ortho-water abundance derived from the SWAS data is only a factor of 5 less than the water abundance derived from ISO.

\subsection{IC $1396 \mathrm{~N}$}

IC $1396 \mathrm{~N}$ is associated with the brightest $\left(L \sim 250 L_{\odot}\right.$, Saraceno et al. 1996) IRAS source $(21391+5802)$ in the IC $1396 \mathrm{H}$ II region (distance 750 pc). It drives an extended bipolar outflow (Codella et al. 2001) and a collimated jet (Nisini et al. 2001).

The ISO-LWS spectrum is rich in $\mathrm{CO}$ and $\mathrm{H}_{2} \mathrm{O}$ lines (Saraceno et al. 2001); the CO lines have been interpreted as due to two gas components: one at $\sim 300 \mathrm{~K}$ and one at $\sim 1400 \mathrm{~K}$, both with a density of $2 \times 10^{6} \mathrm{~cm}^{-3}$. These two physical conditions are both compatible with the observed water line emission, leading to a fractional abundance from $3 \times 10^{-5}$ (at $T=$ $300 \mathrm{~K}$ ) to $\sim 10^{-4}$ (at $T=1400 \mathrm{~K}$ ). Assuming the physical parameters derived from the ISO data in Eq. (1) and the integrated antenna temperature measured by SWAS, we derive an ortho-water fractional abundance of $3 \times 10^{-6}$ at $T=300 \mathrm{~K}$ and $\sim 10^{-5}$ at $T=1400 \mathrm{~K}$, one order of magnitude lower than the ISO abundances. The observed $R(179 / 538)$ is $\sim 10$, lower 
than the theoretical ratio which amounts to 27 and 37 in the models at $T=400 \mathrm{~K}$ and $T=1400 \mathrm{~K}$, respectively. This indicates that the emission of these low energy transitions comes mainly from the colder component of the gas.

\section{4. $L 1157-m m$}

The L1157 dark cloud (distance $440 \mathrm{pc}$ ) is a region of low mass star formation and contains a CO molecular outflow that is associated with an infrared source with a luminosity of $11 L_{\odot}$.

The SWAS beam encompassed almost all the high-velocity $\mathrm{CO}$ emission while ISO mapped the whole outflow by means of three pointings. Very few water and high- $J$ CO lines have been detected in the LWS spectra of L1157-mm outflow (GNL), mainly in the blue lobe. However, rough estimates of the mass, density and temperature in the blue lobe of the outflow ( $M=$ $\left.0.004 M_{\odot}, n\left(\mathrm{H}_{2}\right)=3 \times 10^{5} \mathrm{~cm}^{-3}, T=600 \mathrm{~K}\right)$ can be derived from the high- $J$ CO lines (GNL). Assuming these values in Eq. (1), we derived an ortho-water fractional abundance of $5 \times 10^{-5}$ from the SWAS transition. This is more than one order of magnitude higher than the estimate of NSA. As for NGC 1333-IRAS 4, the discrepancy is due to the higher temperature and lower mass that we have used. Since ISO detected only few water lines, we cannot derive a meaningful water fractional abundance for L1157-mm with the same method used for the other outflows. Alternatively, we note that when the lines are optically thin, as is the case here, the ratio between the $\mathrm{CO}$ and $\mathrm{H}_{2} \mathrm{O}$ abundances is similar to the ratio between their luminosities (Neufeld \& Kaufman 1993). Using this relationship, we derive water fractional abundance of $\sim 5 \times 10^{-5}$, in perfect agreement with the value derived by SWAS. A good agreement is also found between the theoretical and observed $R(179 / 538)$ ratio, 6 and 4 respectively.

\section{Discussion}

In star forming regions, water vapor can be produced by (i) neutral-neutral reactions for gas temperature above $300 \mathrm{~K}$ and (ii) thermal evaporation from icy grain mantles $\left(T_{\text {ev }}\left(\mathrm{H}_{2} \mathrm{O}\right) \geq 90 \mathrm{~K}\right)$. The first channel can be activated behind the front of a non dissociative magneto-hydrodynamical $\mathrm{C}$-shock where water is expected to be the major coolant of the post-shocked gas. In such shocks, dust and gas remain decoupled thus making the thermal ice evaporation very inefficient. However it is also possible that the passage of a relatively slow C-type shock may desorb the mantles, without significant sputtering of the grains. A dust temperature higher than the water ice evaporation temperature can be reached in the inner protostellar envelope. Recent experiments (Fraser et al. 2001) indicate that water ice evaporation should occur at higher temperatures $\left(T_{\text {dust }} \geq 100 \mathrm{~K}\right)$ than previously thought. On the other hand, water vapor can be destroyed by both the UV field generated by fast dissociative J-shocks or by the interstellar UV field in the more external outflow lobes. In the dense envelopes of the very young protostars that we are considering in the paper, the UV field generated by the central object cannot play a significant role.
In Table 2 we show the water fractional abundance derived by ISO and SWAS data. Note that SWAS values (Col. 9) refer only to the ortho form of water. The consistency between the two results is satisfactory if one takes into account that both ISO and SWAS values are affected by large uncertainties. In particular, the SWAS values on the one hand could be underestimated because of the opacity effect of the $538 \mu \mathrm{m}$ line (see Sect. 2); on the other hand the values could be overestimated because the adopted collisional rate may be to low (see Sect. 3). The fractional abundances of water derived from the SWAS data $\left(10^{-5}-10^{-4}\right)$, are higher than previously found by NSA $\left(X\left(0-\mathrm{H}_{2} \mathrm{O}\right) \sim 10^{-6}\right)$; this is because while here we assume that the water is emitted from the same region emitting the high- $J$ CO lines, NSA assume that the fundamental orthowater transition comes from the entire molecular outflow traced by the low- $J$ CO lines and attribute to this region density and temperature derived using other species. In other words, we consider a smaller emitting mass with higher temperature and lower density with respect to NSA. The high spectral and spatial resolution sub-mm data that will be collected by the instruments on board of the Herschel satellite will undoubtedly shed light on the complex structure of the outflow regions and will help constraining their physical conditions.

We note that the theoretical $R(179 / 538)$ derived from the LVG model with the physical conditions traced by the high excitation ISO lines and assuming the same filling factor, is always higher by a factor of 1.5-7 than the observed ratio. Ratios in the range of the observed values (4-10) can be reproduced within the adopted model, only from a gas with $T<$ $500 \mathrm{~K}, n\left(\mathrm{H}_{2}\right)<10^{4} \mathrm{~cm}^{-3}$ and $N\left(\mathrm{o}-\mathrm{H}_{2} \mathrm{O}\right) / \Delta v<10^{15} \mathrm{~cm}^{-2} \mathrm{~km}^{-1} \mathrm{~s}$ (see Fig. 3). However, these values are not realistic because the column density is below the ISO-LWS detection limit. A higher than predicted intensity of the ground state transition of orthowater, detected by SWAS, may indeed be explained if one takes into account that this line can have a contribution from a cooler water component not traced by the transitions available to ISO. These transitions have upper states at energies equivalent to $T \geq 100 \mathrm{~K}$ above the ground state, and consequently they can be significantly populated only at relatively high temperatures.

The possible presence of a cooler gas component can also explain why the fractional abundance derived from the SWAS data is lower than that derived from ISO data. Indeed if the SWAS transition has a contribution in emission from a cooler gas component this should affect the respective estimated ortho- $\mathrm{H}_{2} \mathrm{O}$ fractional abundance by a factor similar to the ratio between the observed and theoretical $R(179 / 538)$ and thus $\lesssim 7$.

A problem remains why the ortho- $\mathrm{H}_{2} \mathrm{O}$ line observed by SWAS towards L1448-mm is so weak with respect to other outflows (such as for example NGC 1333-IRAS 4, which has a water abundance similar to that of L1448-mm) where the transition is so much stronger. A possible explanation may be that the lower $S / N$ for L1448-mm is due to the fact that this outflow has a lower $\mathrm{H}_{2}$ density than the other outflows, in which case a lower $538.3 \mu \mathrm{m}$ flux density is predicted by the LVG model.

While there may be no major discrepancy between the ISO and SWAS outflow observations, the low abundance of water 
in the cold components of the ISM may still not be fully understood: SWAS observations imply gas phase abundances significantly below the values expected from standard models of ion-molecule chemistry in steady-state (e.g. Millar et al. 1997). More recent and complex time-dependent chemical models (e.g. Charnley et al. 2001; Viti et al. 2001; Bergin et al. 2000) propose various solutions that can generally reproduce the gas phase abundances of $\mathrm{H}_{2} \mathrm{O}$ and $\mathrm{O}_{2}$ derived from the SWAS observations. However a common limitation is that they can only do so for a limited period of time, implying recycling of the cloud material on short timescales. For example, some of the conclusions of the Viti et al. (2001) models are that in dense environments $\left(n\left(\mathrm{H}_{2}\right) \geq 10^{3} \mathrm{~cm}^{-3}\right)$ depletion of gas species on to grains must take place and that the lifetime of the clumps of dense gas spans from 1.5 $\mathrm{Myr}$ (for $n\left(\mathrm{H}_{2}\right)=10^{5} \mathrm{~cm}^{-3}$ and a substantial degree of gas depletion) to $6 \mathrm{Myr}$ (for $n\left(\mathrm{H}_{2}\right)=10^{4} \mathrm{~cm}^{-3}$ and weakly bound molecules such as $\mathrm{CO}$ and $\mathrm{N}_{2}$ retained in the gas phase).

Here, we attempt to draw some brief conclusions by looking at the water coming from the cold components in the vicinity of the outflows discussed in this paper. These components must inevitably have been detected by SWAS, considering its large beam. In fact, as one can see from Fig. 1 in this paper and in NSA, there is indeed a self-absorbed component in the emission spectrum of the outflows considered. For this component, the lower limit of the water fractional abundance of the cold gas is $\sim 10^{-9}$ (NSA). However, note that there is great uncertainty in this value as the self-absorption features may not be "pure absorption" and some emission may be present. This will tend to fill in the absorption dip, making it look weaker and resulting in an underestimate of the water abundance in the cold components of the ISM.

In conclusion, we find that there is generally a satisfactory agreement between SWAS and ISO data on the water detected in the four outflows observed by both. The water fractional abundance derived by both satellites is enhanced with respect to that observed in the quiescent gas, and the values derived are consistent with predictions of the shock-chemistry models (Bergin et al. 1998).

Acknowledgements. We acknowledge the referee Prof. E. F. van Dishoeck for her useful comments which have contributed to improve the first version of our manuscript.

\section{References}

Bachiller, R., Chernicharo, J., Martin-Pintado, J., Tafalla, M., \& Lazareff, B. 1990, A\&A, 231, 174

Bergin, E. A., Melnick, G. J., \& Neufeld, D. A. 1998, ApJ, 499, 777

Bergin, E. A., Melnick, G. J., Stauffer, J. R., et al. 2000, ApJ, 539, L129

Ceccarelli, C., Caux, E., Loinard, L., et al. 1999, A\&A, 342, L21

Cernicharo, J., Gonzalez-Alfonso, E., Sempere, M. J., et al. 1999, in The Universe as seen by ISO, ed. P. Cox, \& M. F. Kessler, ESA SP-427, 565

Charnley, S. B., Rodgers, S. D., \& Ehrenfreund, P. 2001, A\&A, 378, 1024

Codella, C., Bachiller, R., Nisini, B., Saraceno, P., \& Testi, L. 2001, A\&A, 376, 271

Draine, B. T., Roberge, W. G., \& Dalgarno, A. 1983, ApJ, 264, 485

Fraser, H. J., Collings, M. P., McCoustra, M. R. S., \& Williams, D. A. 2001, MNRAS, 327, 1165

Giannini, T., Lorenzetti, D., Tommasi, E., et al. 1999, A\&A, 346, 617

Giannini, T., Nisini, B., \& Lorenzetti, D. 2001, ApJ, 555, 40

Green, S., Maluendes, S., \& McLean, A. D. 1993, ApJ, 85, 181

Harwit, M., Neufeuld, D. A., Melnick, G. J., \& Kaufman, M. J. 1998, ApJ, 497, 105

Kaufman, M. J., \& Neufeld, D. A. 1996, ApJ, 456, 611

Melnick, G. J., Ashby, M. L. N., Plume, R., et al. 2000a, ApJ, 539, L87

Melnick, G. J., Stauffer, J. R., Ashby, M. L. N., et al. 2000b, ApJ, 539, L77

Millar, T. J., Farquhar, P. D. R., \& Willacy, K. 1997, A\&AS, 121, 139

Molinari, S., Noriega-Crespo, A., Ceccarelli, C., et al. 2000, ApJ, 538, 698

Neufeld, D. A., \& Kaufman, M. J. 1993, ApJ, 418, 272

Neufeld, D. A., Snell, R. L., Ashby, M. L. N., et al. 2000, ApJ, 539, L107

Nisini, B., Benedettini, M., Giannini, T., et al. 1999, A\&A, 350, 529

Nisini, B., Benedettini, M., Giannini, T., et al. 2000, A\&A, 360, 297

Nisini, B., Massi, F., Vitali, F., et al. 2001, A\&A, 376, 553

Phillips, T. R., Maluendes, S., \& Green, S. 1996, ApJ, 107, 467

Saraceno, P., Ceccarelli, C., Clegg, P., et al. 1996, A\&A, 315, L293

Saraceno, P., Benedettini, M., Codella, C., et al. 2001, in The promise of the Herschel Space Observatory, ed. G. L. Pilbratt, J. Cernicharo, T. Prusti, \& R. Harris, ESA SP-460, 203

Tsuji, T. 2001, A\&A, 376, L1

Truong-Bach, Sylvester, R. J., Barlow, M. J., et al. 1999, A\&A, 345, 925

Viti, S., Roueff, E., Hartquist, T. W., Pineau des Forêts, G., \& Williams, D. A. 2001, A\&A, 370, 557 